\title{
Considerações sobre a função renal no período perioperatório
}

\section{Considerations on renal function in the perioperative period}

ISSN

1809-9475

\author{
Hellen Tatiane de Pontes ${ }^{1}$ \\ Jady Elen de Pontes ${ }^{2}$ \\ Fábio dos Santos Martins Cosso ${ }^{3}$
}

Palavras-chave:

Função renal

Lesão renal aguda

Insuficiência renal aguda

\section{Artigo \\ Original}

Original

Paper

Recebido em 11/2012

Aprovado em 08/2013

\section{Resumo:}

A lesão renal aguda é observada especialmente nas cirurgias de grande porte, em especial nas cirurgias cardíacas, podendo sua prevalência atingir até $40,2 \%$ na população adulta no período pós-operatório imediato. Nesta revisão, buscamos conhecer os aspectos fisiopatológicos envolvidos na lesão renal aguda, os critérios de classificação utilizados atualmente, fatores de risco associados para a disfunção no período pós-cirúrgico imediato e medidas profiláticas. Para tanto, foram pesquisados artigos originais e revisões indexadas a base de dados Medline e Pubmed, compreendendo o período de janeiro de 2002 até junho de 2012, empregando-se os seguintes termos: acute kidney injury, acute renal failure e renal function evaluation. Verificamos que há necessidade de uso de novos biomarcadores associados aos níveis séricos de creatinina e débito urinário atualmente utilizados para o diagnóstico, visto que a detecção precoce da lesão renal aguda por meio da creatinina sérica é difícil de ser estabelecida. Há necessidade de mais estudos que estabeleçam profilaxia farmacológica no tratamento da lesão renal aguda. Medidas como um método padronizado para estratificação de pacientes que apresentam maiores riscos no período pré-operatório, estabelecimento de terapêutica adequada precoce no período perioperatório, introdução nos critérios diagnósticos de novos biomarcadores séricos e urinários serão úteis se utilizados em conjuntos para o manejo clínico adequado nesses pacientes.

\begin{abstract}
:
Acute kidney injury is observed especially in major surgeries, especially cardiac, its prevalence may reach up to $40.2 \%$ in the adult population in the immediate postoperative period. In this review, we seek to understand the pathophysiology involved in acute kidney injury, the classification criteria currently used, risk factors for dysfunction in the immediate postoperative period and prophylactic measures. For that, we researched original articles and reviews indexed in Medline and Pubmed, comprising the period from January 2002 to June 2012, using the following terms: acute kidney injury, acute renal failure and renal function evaluation. We found that there is a need to use new biomarkers associated with creatinine levels and urinary excretion currently used for diagnosis, since early detection of acute kidney injury by serum creatinine is difficult to be established. There is need for further studies to establish pharmacological prophylaxis in the treatment of acute kidney injury. Measures such as a standardized method for stratification of patients presenting risk in higher preoperative, early establishment of appropriate therapy during the perioperative period, the introduction of new diagnostic criteria serum and urine biomarkers will be useful for sets used in the appropriate clinical management in these patients.
\end{abstract}

\section{Keywords}

Renal function

Acute kidney injury

Acute renal failure

\footnotetext{
1 Discente do Curso de Medicina - UniFOA - Centro Universitário de Volta Redonda 


\section{Introdução}

A então denominada Lesão Renal Aguda (LRA) foi descrita, primeiramente, como ischuria renalis por William Heberden, em 1802. Já foi denominada também por Doença aguda de Bright, no início do século XX, e na Primeira Guerra Mundial como "nefrite de guerra", e vem sendo estudada mais do que nunca nos últimos dez anos (EKNOYAN, 2002; DAVES; WELDON apud KIDGO, 2012).

A LRA representa um número de condições que irão afetar a estrutura e a função renal, sendo caracterizada por uma diminuição abrupta dessa função renal. É considerada como uma síndrome clínica de várias etiologias, como, por exemplo, doenças renais específicas (ex.: nefrite intersticial aguda, doenças renais agudas glomerulares e vasculites), condições não específicas (isquemia, lesão por toxicidade) e patologias extra-renais (azotemia pré-renal e nefropatia aguda obstrutiva pós-renal) (KIDGO, 2012).

A LRA pode ser observada no período pós-operatório imediato (POI) de diferentes tipos de cirurgias, sendo as de maior relevância intervenções de grande porte, expondo pacientes ao risco de comprometimento da qualidade de vida, maior tempo de hospitalização, à necessidade de realização de terapia renal substitutiva ou até mesmo à morte (ZACHARIAS et al., 2008; ABELHA et al., 2009; PEREZVALDIVIESO et al., 2009; KOLLI et al., 2010, HOEFIELD et al., 2011).

A disfunção renal pós-operatória é observada em pacientes com função renal preservada antes da cirurgia, bem como em indivíduos com algum grau de comprometimento anterior. Múltiplos fatores podem estar envolvidos no desenvolvimento da LRA no POI, podendo ser devido às condições em que se é submetido durante o procedimento cirúrgico ou por contribuição de condições prévias apresentadas pelo paciente que ofereçam maior risco, somando-se as intervenções intra-operatórias (ZACHARIAS et al., 2008; PEREZ-VALDIVIESO et al., 2009; KOLLI et al., 2010).

A prevalência de LRA no POI pode chegar aproximadamente a $40,2 \%$ na população adulta, dependendo do critério para definir a
LRA e a população estudada, constatando-se as maiores prevalências no POI de cirurgia cardíaca (AZEVEDO et al., 2008; HOSTE et al., 2008; JELDRES et al., 2008; PEREZVALDIVIESO, 2009; KOLLI et al., 2010; CHE et al., 2011).

Evidências sugerem o aumento da mortalidade em indivíduos com comprometimento renal prévio que possuem aumento da creatinina sérica após a cirurgia, bem como daqueles que necessitam de hemodiálise após a evolução para LRA no POI (PEREZ-VALDIVIESO et al., 2009; KOLLI et al., 2010).

Embora na literatura médica estejam descritos cerca de 35 procedimentos para avaliação da função renal, os estudos mais recentemente publicados vêm empregando o critério Acute Kidney Injury Network (AKIN), uma modificação do score RIFLE, validado em 2007. No critério AKIN, são levados em consideração os níveis séricos de creatinina e débito urinário, podendo-se classificar o paciente quanto ao grau de severidade da lesão em três níveis. Atualmente, a iniciativa KIDGO (Kidney Disease: Improving Global Outcomes) propôs pequenas alterações no critério AKIN (BELLOMO et al., 2007; METHA et al., 2007; AZEVEDO et al., 2008; JELDRES et al., 2008; ALKHUNAIZI et al., 2011; CHE et al., 2011; KDIGO, 2012).

O desenvolvimento de um método de pontuação prognóstica padronizado para o desenvolvimento de IRA no POI, como, por exemplo, o projeto AKICS score para pacientes de cirurgia cardíaca, seria útil para identificar os indivíduos com risco potencial visando a desenvolver essa complicação e estabelecer tratamento precoce (PALOMBA et al., 2007).

Segundo Hoefield et al. (2011), nos dias de hoje não existe nenhuma terapia farmacológica específica para o tratamento da LRA. No entanto, é possível identificar os pacientes sob risco para o seu desenvolvimento, tornando possível a implementação de medidas profiláticas que minimizem os riscos.

A seguir, abordaremos os aspectos fisiopatológicos envolvidos na IRA, critérios de classificação utilizados atualmente, fatores de risco associados para a disfunção renal no período pós-cirúrgico imediato e medidas de prevenção para seu desenvolvimento. 


\section{Metodologia}

Para construção desta revisão, foram pesquisados artigos originais e revisões indexadas na base de dados Medline e Pubmed, compreendendo o período de janeiro de 2002 até junho de 2012 empregando-se os seguintes termos: acute kidney injury, acute renal failure e renal function evaluation.

\section{Revisão de Literatura}

Dada a participação dos rins na manutenção do equilíbrio hidroeletrolítico, excreção de produtos do metabolismo e compostos estranhos, em seu papel endócrino, na regulação da pressão arterial, manutenção do equilíbrio ácido-básico dentre outras funções, eis a importância da preservação da função normal desse órgão (GUYTON; HALL, 2011).

A LRA é uma desordem complexa que pode ocorrer em uma variedade de situações, com expressões clínicas que podem variar desde um leve aumento na creatinina sérica até insuficiência renal anúrica, com diminuição abrupta da função renal (METHA et al., 2007; BONVENTE; YANG, 2011).

$\mathrm{Na}$ insuficiência renal aguda ocorrem danos à capacidade de excreção renal e da manutenção do equilíbrio hidroeletrolítico, bem como acúmulo de uréia e creatinina, podendo ou não ocorrer diminuição do débito urinário (AZEVEDO et al. 2008).

A redução do fluxo sanguíneo renal local ou generalizada representa a fisiopatologia da LRA, podendo ser ocasionada em diversos estados fisiopatológicos e por medicamentos. Alguns dos acontecimentos que podem levar a essa circunstância são os seguintes: síndrome hepatorrenal, depleção de volume intravascular e hipotensão, diminuição efetiva do volu- me intravascular, medicamentos, sepse, doença vascular renal, dentre outros. Para todos esses fatores, a isquemia renal é a via comum e fator determinante para a manifestação da LRA (BONVENTE; YANG, 2011).

A função renal é mais satisfatoriamente avaliada por meio da estimativa do ritmo de filtração glomerular (RFG). Diversos métodos bioquímicos estão disponíveis atualmente, para avaliação da função renal por meio da estimativa do RFG (KIDGO, 2012).

A IRA pode ser definida baseando-se num dos critérios descritos a seguir (METHA et al., 2007):

- $\quad$ aumento da creatinina sérica $\geq 0,3 \mathrm{mg} / \mathrm{dL}$ ( $\geq 26 \mu \mathrm{mol} /$ litro) dentro de 48 horas;

- $\quad$ aumento da creatinina sérica $\geq 50 \%(1,5$ vezes a partir de um valor referência previamente conhecido;

- débito urinário $<0,5 \mathrm{~mL} / \mathrm{Kg} / \mathrm{h}$ por mais de 6 horas consecutivas.

O valor de creatinina sérica utilizado como referência deverá ser o menor padrão mensurado nos últimos três meses e, no que tange ao método de detecção de lesão renal aguda por diminuição do débito urinário, esse deve ser utilizado com cautela, pois sua mensuração fora das unidades de cuidado intensivo pode não ser confiável, em consequência da possibilidade de ocorrência de lesão renal com oligúria e sem oligúria, sendo então, para tanto, importante o exame clínico (HOEFIELD et al., 2011).

Após determinação do estabelecimento da lesão renal, deve-se estratificar o paciente quanto ao grau de dano, exemplificado na tabela 1. Os dados fornecidos por essa tabela são modificações do critério Acute Kidney Injury Network (AKIN) para estratificação da lesão renal, propostos pela fundação "Kidney Disease: Improving Global Outcomes" (KDIGO) (METHA et al., 2007; KDIGO, 2012).

\section{Tabela 1 - Classificação da lesão renal aguda}

$\begin{array}{ll}\text { Estágio } & \text { Creatinina Sérica } \\ 1 & \uparrow \text { Creatinina } \geq 26 \mu \mathrm{mol} / \text { litro ou } \\ & \uparrow \text { Creatinina } \geq 1,5-1,9 \text { vezes desde o início } \\ 2 & \uparrow \text { Creatinina } \geq 2-2,9 \text { vezes desde o início } \\ 3 & \uparrow \text { Creatinina } \geq 3 \text { vezes desde o início ou } \\ & \uparrow \text { Creatinina de } 354 \mu \mathrm{mol} / \text { litro ou terapia renal } \\ & \text { substitutiva, independente da fase }\end{array}$

\section{Débito Urinário}

$<0,5 \mathrm{~mL} / \mathrm{Kg} /$ Hora por mais que 6 horas consecutivas

$$
\begin{aligned}
& <0,5 \mathrm{~mL} / \mathrm{Kg} / \text { Hora por mais que } 12 \text { horas } \\
& <0,3 \mathrm{~mL} / \mathrm{Kg} / \text { Hora por mais que } 24 \text { horas ou anúria } \\
& \text { por } 12 \text { horas }
\end{aligned}
$$


A prevenção da lesão renal deve ser considerada em duas instâncias: primeiro, deve-se evitar a ocorrência da lesão e, em segundo lugar, prevenir a progressão após lesão já estabelecida (HOEFIELD et al., 2011).

Os fatores de risco para a disfunção renal são de natureza multifatorial, a considerar: idade $>75$ anos, doença renal crônica prévia em estágio $3\left(\mathrm{RFG}<60 \mathrm{~mL} / \mathrm{min} / 1.73 \mathrm{~m}^{2}\right)$, doença hepática, diabetes mellitus, insuficiência cardíaca, doença aterosclerótica vascular periférica, rabdomiólise, uso de fármacos nefrotóxicos, hipotensão, sepse, hipovolemia, administração de contrastes (pouco importante em indivíduos sem lesão renal prévia) e indução anestésica (ZACHARIAS et al., 2008; HOEFIELD et al., 2011).

Atenção especial deve ser dada a população idosa, que está mais propensa a apresentar disfunção renal pós-operatória devido, especialmente, a três fatores: pela diminuição da função renal devido à idade, por serem portadores de comorbidades que se somam para o prejuízo da função renal e uso de diversos medicamentos (AZEVEDO et al., 2008; MODIG et al., 2011).

A indução anestésica levando a uma hipotensão e predispondo o paciente a uma isquemia renal, somando-se ao procedimento cirúrgico em si (alguns apresentam maiores riscos), com grandes perdas sanguíneas podem contribuir para o prejuízo da função renal, bem como por fatores prévios do paciente (AZEVEDO et al., 2008; ZACHARIAS et al., 2008).

A exposição a alguns fatores pode desencadear o desenvolvimento da LRA, tais como os seguintes (KIDGO, 2012):

- Sepse

- Doença crítica

- Choque circulatório

- Queimaduras

- Trauma

- Cirurgia cardíaca (especialmente com circulação extracorpórea)

- Cirurgia grande não cardíaca

- Drogas nefrotóxicas

- Agentes de radiocontraste

- Plantas e animais venenosos
Alguns indivíduos, e algumas condições desses, também são mais susceptíveis e devem ser mais monitorados (KIGDO, 2012):

- Desidratação e depleção de volume

- Idade avançada

- Sexo feminino

- Raça negra

- Doença renal crônica

- Doenças crônicas (cardíacas, pulmonares, hepáticas)

- Diabetes mellitus

- Câncer

- Anemia

De acordo com Zacharias et al. (2008), não existem evidências claras na literatura de alguma medida eficaz para proteção renal durante a cirurgia. Em sua revisão intitulada "Intervenções para proteção renal no período perioperatório" foram verificadas a eficácia de várias medidas preconizadas para proteger os rins no período perioperatório.

A lesão renal aguda tem como consequências alterações nos líquidos corporais, eletrólitos, equilíbrio ácido base e regulação hormonal, resultando em anormalidades no sistema nervoso central, imune e de coagulação (KIDGO, 2012).

\section{Resultados e Discussão}

Pequenas elevações nos níveis séricos e creatinina podem estar associados à mortalidade. No entanto, suas explicações não estão totalmente claras, podendo ser devido aos efeitos adversos da função renal diminuída, como, por exemplo, sobrecarga de volume, retenção de corpos urêmicos, acidose, distúrbios eletrolíticos, aumentos do risco de infecção e anemia (KIDGO, 2012).

No estudo de Che et al. (2010), o uso de cinco biomarcadores séricos e urinários para a detecção precoce de LRA em pacientes submetidos a cirurgia cardíaca eletiva, se mostraram úteis em detrimento dos parâmetros baseados apenas nos níveis séricos de creatinina. 
Outros estudos tentam avaliar de diversas maneiras a função renal com objetivo de detecção, o mais precocemente possível, por meio de diversos biomarcadores, tais como Cistatina- $C$, níveis urinários de neutrófilos gelatinase associada lipolina (NGAL), interleucina-18 (IL-18), proteína de ligação do retinol (RBP), N-acetil- $\beta$-glicosaminidase (NAG), a1-microglobulina, sE-selectina, sICAM-1, oxigênio estável e isótopos de hidrogênio no plasma, dentre outros (DEHNE et al., 2008, EAGLESTONE; ELLIS, 2009; CHE et al., 2010; KIDGO, 2012; KUO et al., 2012).

A taxa de filtração glomerular, entretanto, é mais amplamente aceita como o melhor indício da função renal, embora essa seja estimada a partir de marcadores endógenos filtrados, como a creatitina. Porém, sabe-se que a detecção precoce da LRA e a maioria dos casos somente são diagnosticados após aumentos significativos na creatinina sérica (KIDGO, 2012).

Marcadores tais como o débito urinário, em algumas situações, podem não ser confiáveis, tais como a sepse, que é a condição mais comum associada à LRA em unidades de terapia intensiva, que pode alterar a função renal sem gerar alterações características nos índices de urina (KIDGO, 2012).

Para Lassnigg et al. (2004), nos pacientes submetidos a cirurgia cardíaca, um aumento dos níveis de creatinina $\geq 0,5 \mathrm{mg} / \mathrm{dL}(\geq 44,2$ $\mu \mathrm{mol} / \mathrm{L})$ ou uma diminuição $>0,3 \mathrm{mg} / \mathrm{dL}(>$ $26,5 \mu \mathrm{mol} / \mathrm{L})$ foi associado com pior sobrevida, diferentemente dos padrões estabelecidos para a classificação da LRA.

Múltiplas definições são descritas na literatura, levando a diferentes incidências e taxas de mortalidades nos pacientes, porém, a definição mais amplamente aceita surgiu a partir do critério RIFLE, que passou, nos últimos anos, por algumas modificações.

Foi comprovado que, quando rins de mamíferos são submetidos à isquemia quente e por período prolongado, seguindo-se de reperfusão, ocorre necrose extensiva e destruição dos túbulos proximais na parte externa da medula renal (KIDGO apud SHANLEY et al., 1986), podendo explicar possivelmente lesões renais por isquemia nas grandes cirurgias.

No estudo de Feng et al. (2010), a técnica de baixa pressão venosa central com o objetivo de diminuir perdas sanguíneas não prejudicou a função renal em pacientes submetidos a transplante de fígado em relação a pacientes submetidos a transplante por meio de técnica usual.

De acordo com Fischer et al. (2002), apenas os pacientes com função renal normal no período operatório que tiveram cirurgias com menor tempo de duração em circulação extracorpórea apresentaram função renal normal no período pós-operatório em relação àqueles em iguais condições que permaneceram em circulação extracorpórea por tempo superior. Os pacientes que permaneceram em maior tempo com pressão arterial média $<60 \mathrm{mmHg}$ apresentaram disfunções renais pós-operatórias.

Notamos que a maioria das publicações relacionadas a procedimentos cirúrgicos e associação com LRA abrange mais frequentemente as cirurgias cardíacas. Poucos estudos falam da associação entre outros procedimentos cirúrgicos mais comuns e frequentes e LRA.

Em suma, para a redução da morbidade e mortalidade por LRA pós-operatória é importante o desenvolvimento de vários métodos em conjunto para seu manejo clínico adequado, a considerar-se: um método padronizado para estratificação de pacientes que apresentam maior risco no período pré-operatório, estabelecimento de terapêutica adequada precoce no período perioperatório, introdução nos critérios diagnósticos de novos biomarcadores séricos e urinários que permitam a identificação precoce de LRA.

\section{Referências}

1. EKNOYAN G. Emergence of the concept of acute renal failure. American Journal Nephrology, v. 22, p. 255-230, 2002.

2. Kidney Disease: Improving Global Outcomes (KIDGO) Acute Kidney Injury Work Group. KIDGO Clinical Practice Guideline for Acute Kidney Injury. Kidney International., v. 2, p. 1-38, 2012.

3. ZACHARIAS M., CONLON N.P., HERBISON G.P., SIVALINGAM P., WALKER R.J., HOVHANNISYAN K. Interventions for protecting renal function in the perioperative period (Review). The

Cochrane Library, v. 4, 2008. 
4. ABELHA F.J., BOTELHO M., FERNANDES V., BARROS H. Outcome and quality of life of patients with acute kidney injury after major surgery. Nefrologia, v. 29, p. 404-414, 2009.

5. PEREZ-VALDIVIESO

J.R., MONEDERO P., VIVES M., GARCIAFERNANDEZ N., BES-RASTROLLO M. Cardiac-surgery associated acute kidney injury requiring renal replacement therapy. A Spanish retrospective case-cohort study. BMC Nephrology, v. 10, p. 1-10, 2009.

6. KOLLI H., RAJAGOPALAM S., PATEL N., RANJAN R., VENUTO R., LOHR $\mathrm{J}$, et al. Mild acute kidney injury is associated with increased mortality after cardiac surgery in patients with eGFR $<60 \mathrm{~mL} / \mathrm{min} / 1.73 \mathrm{~m}^{2}$. Renal Failure, v.32, p. 1066-1072, 2010.

7. HOEFIELD R., POWER A., WILLIAMS N., LEWINGTON A.J.P. Preventing acute kidney injury: identifying risk and reducing injury. British Journal of Hospital Medicine, v. 72, n. 9, p. 492-6, 2011.

8. AZEVEDO V.L.F., SILVEIRA M.A.S., SANTOS J.N., BRAZ J.R.C., BRAZ L.G., MÓDOLO N.S.P. Postoperative renal function evaluation, through RIFLE criteria, of elderly patients Who underwent femur fracture surgery under spinal anesthesia. Renal Failure, v. 30, p. 485-490, 2008.

9. HOSTEE.A.,CRUZD.N., DAVENPORT A., MEHTA R.L., PICCINNI P., TETTA C., et al. The epidemiology of cardiac surgery-associated acute kidney injury. The International Journal of Artificial Organs, v. 31, p. 158-165, 2008.

10. JELDRES C., BENSALAH K., CAPITANIO U., ZINI L., PERROTE P., SUARDI N., et al. Baseline function renal, ischaemia time and blood loss predict the rate of renal failure after partial nephretomy. Journal Compilation, v. 103, p. 1632-1635, 2008.
11. CHE M., LI Y., LIANG X., XIE B., XUE S., QIAN J., et al. Prevalence of acute kidney injury following cardiac surgery and related risk factors in Chinese patients. Nephron Clinical Practice, v. 117, p. 305-311, 2011.

12. BELLOMO R., KELLUM J.A., RONCO C. Defining and classifying acute renal failure: from advocacy to consensus and validation of the RIFLE criteria. Intensive Care Med, v. 33, p. 409-413, 2007.

13. METHA R.L., KELLUM J.A., SHA S.V., MILITORIS B.A., RONCO C., WARNOCK D.G., et al. Acute Kidney Injury Network: report of an initiative to improve outcomes in acute kidney injury. Critical Care, v. 11, p. 1-8, 2007.

14. ALKHUNAIZI A.M., SHAH S.S.A., WESSLEN U.S., AL SADAH Z.A., ANTONY A. Acute kidney injury after cardiac surgery in eastern Saudi Arabia. Eastern Mediterranean Health Journal, v. 17, p. 495-500, 2011.

15. PALOMBA H., CASTRO I., NETO A.L.C., LAGE S., YU L. Acute kidney injury prediction following elective cardiac surgery: AKICS score. Kidney International, v. 72, p. 624-631, 2007.

16. GUYTON, A.C., HALL J. Tratado de Fisiologia Médica. Tradução de Barbara de Alencar Martins et al. Rio de Janeiro: Elsevier, p. 961-977, 2006.

17. BONVENTRE J.V., YANG L. Cellular pathophysiology of ischemic acute kidney injury. American Society for Clinical Investigation, v. 121, n. 11, p. 4210-4221, 2011.

18. MODIG S., LANNERING C., ÖSTGREN C.J., MÖLSTAD S., MIDLÖV P. The assessment of renal function in relation the use of drugs in elderly in nursing homes; a cohort study. BMC Geriatrics, v. 11, p. 1-6, 2011. 
19. CHE M., XIE B., XUE S., DAI H., QIAN J., NI Z., et al. Clinical Usefulness of Novel Biomarkers for the detection of acute kidney injury following elective cardiac surgery. Nephron Clinical Practice, v. 115, p. 66-72, 2010.

20. DEHNE M.G., SABLOTZKI A., MÜHLING J.; et al. Evaluation of sESelectin and sICAM-1 as Parameters for Renal Function. Renal Failure, v. 30, p. 675-684, 2008.

21. EAGLESTONE G., ELliS P. Cystatin $\mathrm{C}$ as a marker of renal function in older adults. Art \& Science, v. 24, n. 13, p. 359, 2009.

22. KUO T-C., WANG C-H., LIN H-C., LIN Y-H., LIN M., et al. Assessment of Renal Function by the Stable Oxygen and Hydrogen Isotopes in Human Blood Plasma. PLoS ONE, v. 7, n. 2, p. 1-9, 2012.
23. LASSNIG A., SCHMIDLIN D., MOUHIEDDINE, et al. Minimal changes of serum creatinine predict prognosis in patients after cardiothoracic surgery: a prospective cohort study. Journal of the American Society of Nephrology, v. 15, p. 1597-1605, 2004.

24. FENG Z-Y., XU X., ZHU S-M., BEIN B., ZHENG S-S. Effects of law central venous pressure during preanhepatic phase on blood loss and liver and renal function in liver transplantation. World Journal of Surgery, v. 34, p. 1864-1873, 2010.

25. FISCHER U.M., WEISSENBERGER W.K., WARTERS R.D., GEISSLER H.J., ALLEN S.J., MEHLHORN U. Impact of cardiopulmonary bypass management on postcardiac surgery renal function. Perfusion, v. 17, p. 401-6, 2002. 